OPEN ACCESS

Edited by:

Ovidiu Constantin Baltatu

Camilo Castelo Branco University,

Brazil

Reviewed by:

Masaki Mizuno,

University of Texas Southwestern Medical Center, USA

Takatoshi Kasai,

Juntendo University, Japan

*Correspondence:

Hisayoshi Murai

sakurasoma1209@yahoo.co.jp (HM)

Specialty section:

This article was submitted to Integrative Physiology, a section of the journal

Frontiers in Physiology

Received: 10 December 2015 Accepted: 12 February 2016 Published: 02 March 2016

Citation:

Hamaoka T, Murai H, Kaneko S, Usui S, Okabe Y, Tokuhisa H, Kato T, Furusho $H$, Sugiyama $Y$, Nakatsumi $Y$,

Takata S and Takamura M (2016) Single-Unit Muscle Sympathetic Nerve Activity Reflects Sleep Apnea Severity, Especially in Severe Obstructive Sleep Apnea Patients. Front. Physiol. 7:66.

doi: 10.3389/fphys.2016.00066

\section{Single-Unit Muscle Sympathetic Nerve Activity Reflects Sleep Apnea Severity, Especially in Severe Obstructive Sleep Apnea Patients}

\author{
Takuto Hamaoka ${ }^{1}$, Hisayoshi Murai ${ }^{1 *}$, Shuichi Kaneko ${ }^{1}$, Soichiro Usui ${ }^{1}$, Yoshitaka Okabe ${ }^{1}$, \\ Hideki Tokuhisa ${ }^{1}$, Takeshi Kato ${ }^{1}$, Hiroshi Furusho' ${ }^{1}$, Yu Sugiyama ${ }^{2}$, Yasuto Nakatsumi ${ }^{2}$, \\ Shigeo Takata ${ }^{2}$ and Masayuki Takamura ${ }^{1}$ \\ ' Department of Disease Control and Homeostasis, Graduate School of Medical Science, Kanazawa University, Kanazawa, \\ Japan, ${ }^{2}$ Kanazawa Municipal Hospital, Kanazawa, Japan
}

Obstructive sleep apnea syndrome (OSAS) is associated with augmented sympathetic nerve activity, as assessed by multi-unit muscle sympathetic nerve activity (MSNA). However, it is still unclear whether single-unit MSNA is a better reflection of sleep apnea severity according to the apnea-hypopnea index (AHI). One hundred and two OSAS patients underwent full polysomnography and single- and multi-unit MSNA measurements. Univariate and multivariate regression analysis were performed to determine which parameters correlated with OSAS severity, which was defined by the AHI. Single- and multi-unit MSNA were significantly and positively correlated with AHI severity. The AHI was also significantly correlated with multi-unit MSNA burst frequency $(r=0.437, p<0.0001)$ and single-unit MSNA spike frequency $(r=0.632, p<0.0001)$. Multivariable analysis revealed that SF was correlated most significantly with $\mathrm{AHI}(T=$ $7.27, p<0.0001)$. The distributions of multiple single-unit spikes per one cardiac interval did not differ between patients with an $\mathrm{AHI}$ of $<30$ and those with and $\mathrm{AHI}$ of 30-55 events/h; however, the pattern of each multiple spike firing were significantly higher in patients with an $\mathrm{AHI}$ of $>55$. These results suggest that sympathetic nerve activity is associated with sleep apnea severity. In addition, single-unit MSNA is a more accurate reflection of sleep apnea severity with alternation of the firing pattern, especially in patients with very severe OSAS.

Keywords: microneurography, sleep apnea syndromes, muscle sympathetic nerve activity, single-unit recordings, apnea-hypopnea index

\section{INTRODUCTION}

Obstructive sleep apnea syndrome (OSAS) is a known risk factor for cardiovascular events (Mooe et al., 1996), and increased sympathetic nerve activity can worsen the mortality and severity of both cardiovascular disease and OSAS. Several mechanisms are hypothesized to increase sympathetic nerve activity in OSAS patients. In patients with OSAS, blood oxygenation levels decrease during sleep, and carbon dioxide levels increase as a result, and these reactions stimulate peripheral chemoreceptors to increase central sympathetic nerve activity (Leuenberger et al., 1995; Morgan et al., 1995). In addition, afferent vagal signals from pulmonary stretch receptors are diminished, 
resulting in augmented sympathetic nerve activity (Bradley et al., 2003). The decrease in blood pressure and oxygenation also shift the set point of the arterial baroreceptors to the high pressure range, and as a result, blood pressure is increased both during the day and night (Cooper et al., 2005, 2007). These results suggest that decreasing sympathetic nerve activity and improving hypoxia are both important when treating OSAS.

To assess sympathetic nerve activity, muscle sympathetic nerve activity (MSNA), a direct recording of efferent sympathetic nerve activity, is still recognized as the gold standard method (Vallbo et al., 1979). Multi-unit MSNA frequency (BF) and incidence (BI) are generally used to assess sympathetic nerve activity quantitatively; however, the multi-unit MSNA index may underestimate the actual degree of central sympathetic nerve signaling to peripheral organs. Recently, we demonstrated that single-unit MSNA is more accurate than multi-unit MSNA, especially in intense sympathoexcitation states such as heart failure (Murai et al., 2006, 2009; Ikeda et al., 2012). Elam et al. (2002) reported that the number of multiple firings during one cardiac interval in OSAS patients was significantly increased compared with systolic heart failure (HF) patients, although multi-unit MSNA levels were similar between groups. Despite the interesting results from this study, only eight OSAS patients were included, so interpreting the relationship between sleep parameters and single-unit MSNA may be hard.

The apnea-hypopnea index (AHI), which is used to grade the severity of apnea in OSAS patients, is strongly related to mortality risk (Young et al., 2008). To date, little research has been performed using multi- and single-unit MSNA to examine the relationship between AHI and sympathetic nerve activity in OSAS patients. In addition, the degree to which the firing characteristics of central sympathetic nerve activity to the periphery is associated with AHI severity is unclear. In this study, we hypothesized that single-unit MSNA assessment in OSAS patients would provide more accurate information regarding disease severity compared with multi-unit MSNA.

\section{METHODS}

\section{Subjects}

OSAS was diagnosed in patients with an AHI of $\geq 15$ or $\geq 5$ with daytime sleepiness. Patients who had more than five central sleep apnea events per hour were excluded. Patients were also excluded if they had HF, unstable angina pectoris, myocardial infarction, and/or coronary revascularization within 4 weeks of the study. HF was defined in accordance with the American Heart Association/American College of Cardiology guidelines (Hunt et al., 2009). Briefly, HF was diagnosed if patients had a left ventricular ejection fraction (LVEF) of $\leq 40 \%$

Abbreviations: AHI, apnea-hypopnea index; AUC, area under the curve; BF, burst frequency; BI, burst incidence; BMI, body mass index; ESS, Epworth sleepiness scale; MSNA, muscle sympathetic nerve activity; PSG, polysomnography; ROC, receiver operating characteristics curve; SES, sleep efficiency score; SF, spike frequency; S group, SAS group; SI, spike incidence; SREM, stage rapid eye movement; SS group, severe SAS group; VSS group, very severe SAS group. despite optimal treatment for at least 1 month or an LVEF of $>40 \%$ with a history of acute pulmonary edema after excluding other non-cardiogenic diseases. Patients with atrial fibrillation or any implanted pacemaker devices, including implantable cardioverter defibrillators or cardiac resynchronization therapy, were excluded. Patients with an estimated glomerular filtration rate of $<30 \mathrm{ml} / \mathrm{min} / 1.73 \mathrm{~m}^{2}$ were also excluded. These conditions can affect sympathetic nerve activity (Hausberg et al., 2002; Hogarth et al., 2006).

This protocol was approved by the Research Ethics Board of the University of Kanazawa (Kanazawa, Japan). This study has been registered in the University Hospital Medical Information Network Center (UMIN, TOKYO, Japan) Clinical Trials Registration System as UMIN000017612. All patients provided informed written consent.

\section{Experimental Protocols}

This study was designed as a cross-sectional, observational trial. All investigators that assessed MSNA and PSG data were blinded to each patient's severity and characteristics.

\section{Polysomnography}

PSG monitoring was performed overnight in the Sleep Disorders Laboratory of Kanazawa municipal hospital using an Embla N7000 system (Natus, San Carlos, CA, USA). Electroencephalogram (R\&K method), right and left electrooculogram, body position, thoracic, and abdominal wall motion (Respiratory Inductive Plethysmogram system), electrocardiogram, or nasal airflow (Thermistor and pressure sensor), and oxygen saturation, measured using a finger probe pulse oximeter (Nonin 8000J Adult Flex Sensor, Plymouth, MN, USA), and the patient's sleep state were recorded during the session. Experienced investigators subsequently analyzed these data using Rembrandt analysis software (Natus, San Carlos, CA, USA). This inspection method was included in the type 1 category of the American Academy of Sleep Medicine, American Thoracic Society and the American College of Chest Physicians manuals for the examination of suspected sleep apnea in adults (Chesson et al., 2003). Patients were admitted to the laboratory at 16:00 on the day of measurement, and preparation for PSG started at 20:00. Measurement started at 21:00 after the lights were turned off and continued till 6:00 the next morning. All polysomnography measurements were supervised by a physician who was registered as a polysomnographic technologist by the American Academy of Sleep Medicine and a physician certified in sleep medicine by the Japanese Society of Sleep Research. Technicians were also qualified and certified.

\section{Apnea Hypopnea Index}

The AHI was determined using standard criteria, which are described briefly below (Berry et al., 2012). An apnea event was scored when both of the following conditions were met: (1) a $\geq 90 \%$ drop in the peak signal, measured by an oronasal thermal sensor, compared with pre-event baseline levels (2) that was $\geq 10 \mathrm{~s}$ in duration. Apnea events with a long duration or increased inspiratory effort were defined as obstructive apnea events, and lack of inspiratory effort during the apnea event was defined 
as a central apnea event. If inspiratory effort was diminished and resumed later in the apnea event, it was scored as a mixed apnea event. A hypopnea event was scored if all of the following were met: (1) the peak signal excursions dropped by $\geq 30 \%$ of pre-event baseline using nasal pressure, (2) the duration of the $\geq 30 \%$ drop in signal excursion was $\geq 10$ s, and (3) there was $\geq 3 \%$ oxygen desaturation from pre-event baseline or the event was associated with an arousal. Obstructive hypopnea was defined when at least one of the following was met during a hypopnea event: (1) snoring, (2) flattening of the nasal pressure, or (3) paradoxical motion of the chest and abdominal band excursions on respiratory inductance plethysmography. Hypopnea events that lacked these characteristics were defined as central hypopnea events. The AHI was defined as the number of episodes of apnea and hypopnea per hour of sleep.

\section{Muscle Sympathetic Nerve Activity}

MSNA recordings were taken in patients who had been diagnosed with OSAS within 1 week after polysomnography. All data were collected in the morning at 10:00. All participants were asked to abstain from alcohol and caffeine for $24 \mathrm{~h}$ and were tested at least $12 \mathrm{~h}$ post-prandial. Postganglionic MSNA was recorded from the right peroneal nerve at the fibular head using a high-impedance (10 M $\Omega$ ) tungsten microelectrode. As described previously (Murai et al., 2006, 2009; Ikeda et al., 2012; Millar et al., 2015), the common peroneal nerve was detected by palpation and stimulated electrically at the skin surface. Investigators inserted a tungsten microelectrode percutaneously into a motor fascicle of the peroneal nerve. The microelectrode was adjusted until spontaneous pulse-synchronous, multi-unit bursts of sympathetic nervous activity could be validated. The microelectrode was adjusted further until a large unitary spike could be distinguished from background noise in the recording, which allowed for single-unit MSNA analysis. Single- and multiunit MSNAs were recorded simultaneously from the same microelectrode. After a 15-min stabilization period, data were acquired over at least $5 \mathrm{~min}$.

The electrodes were connected to a preamplifier at a gain of 1000 and to an amplifier at a gain of 70. The signal was fed through a band-pass filter $(500-3000 \mathrm{~Hz})$ and a resistancecapacitance integrated circuit with a time constant of $0.1 \mathrm{~s}$ to produce a mean voltage neurogram using a Power Lab recoding system (Model ML 785/85P; ADI Instruments, Bella Vista, Australia). The raw nerve signal was obtained at $12 \mathrm{kHz}$; other signals were obtained at $1000 \mathrm{~Hz}$.

Once offline, experienced investigators identified multi-unit MSNA peaks in the integrated nerve recording based on the relationship with cardiac activity in a blinded fashion. Multi-unit

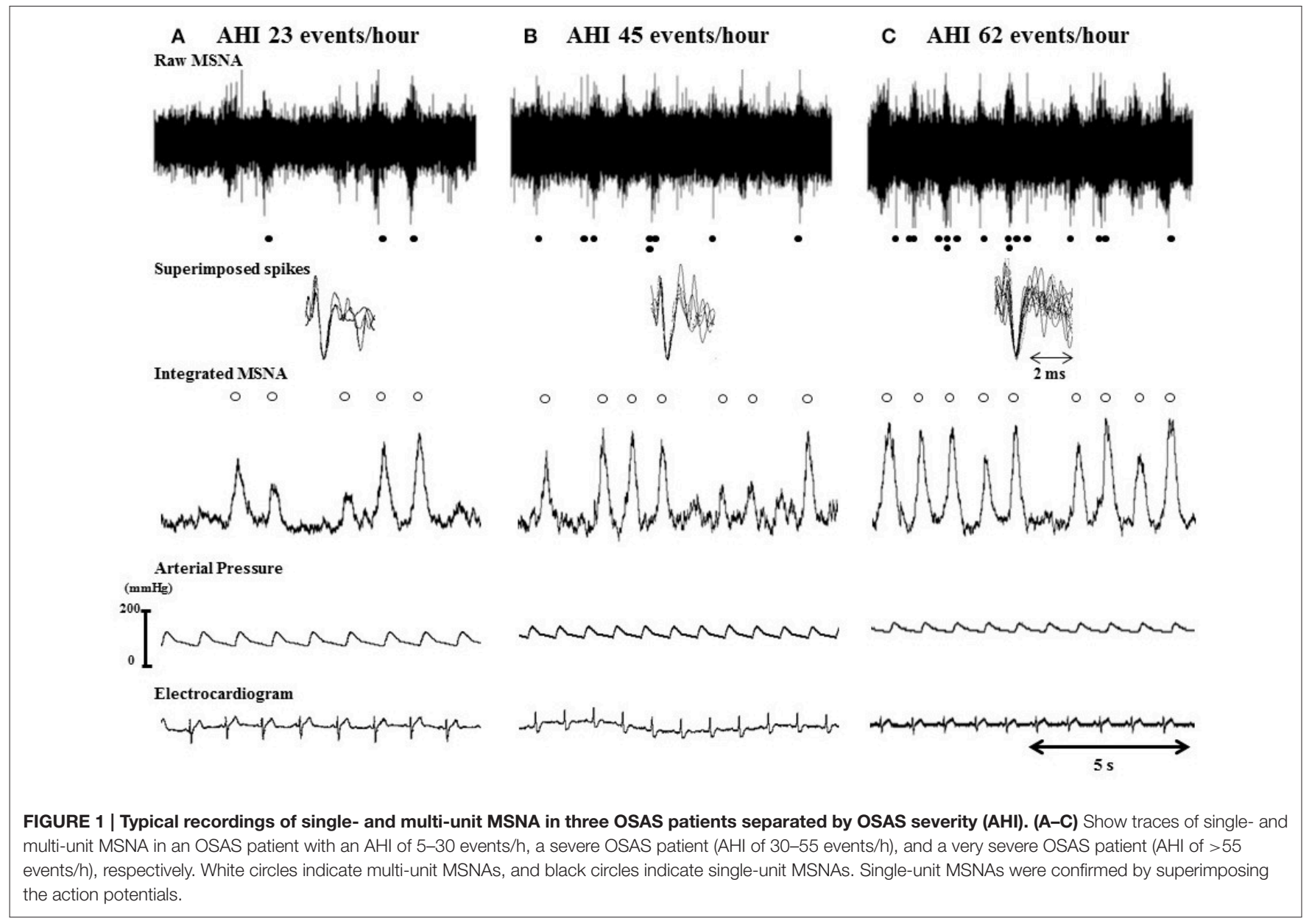


MSNA was expressed as the number of bursts per minute (burst frequency: BF) and the number of bursts per 100 heartbeats (burst incidence: $\mathrm{BI}$ ).

Figure 1 shows typical single- and multi-unit integrated nerve recordings from OSAS patients divided by OSAS severity (AHI). When the raw neurogram record was clear enough to identify single-unit MSNA spikes, spike morphology was carefully inspected by experienced investigators. Single-unit spikes were defined as (1) spike synchronization with multi-unit MSNA bursts, (2) triphasic spike morphology with a negative main phase, and (3) superimposition of candidate action potentials with minimal variation. Single-unit MSNA was expressed as the number per minute (spike firing frequency: SF) and the number per 100 heartbeats (spike firing incidence: SI). Firing probability was defined as the percentage of heartbeats during which one or more spikes occurred. The firing probability was calculated from the number of cardiac intervals showing single-unit spikes divided by all cardiac intervals. The probability of multiple spikes was calculated from the number of cardiac intervals in which multiple spikes (two, three, or four single-unit spikes) were fired divided by all cardiac intervals with at least one spike. In addition, the percentage of cardiac intervals showing one, two, three, or four single-unit spikes was calculated from the number of cardiac intervals in which each single-unit spike were fired divided by all cardiac intervals with at least one spike. In this study, four was the maximum number of single-unit MSNA spikes during one cardiac cycle, which was the same as our previous study and the same as others have reported (Elam et al., 2002; Murai et al., 2012). The inter- and intra-observer correlations of multiunit MSNA in this study were 0.890 and 0.91 , respectively $(p<$ $0.001)$ and for single-unit MSNA were 0.88 and $0.91(p<$ 0.001 ). The reproducibility of single-unit MSNA has also been reported in previous reports (Lambert et al., 2011, Hering et al., 2013).

\section{Statistical Analysis}

All data are presented as means \pm SD. Statistical analysis was performed using SPSS for Windows (version 17.0; SPSS Japan Inc. Tokyo, Japan) and EZR (Saitama Medical Center, Jichi Medical University, Saitama, Japan), which is a graphical user interface for $\mathrm{R}$ (The R Foundation for Statistical Computing, Vienna, Austria). More precisely, it is a modified version of $\mathrm{R}$ commander designed to add statistical functions frequently used in biostatistics. Univariate regression analysis was performed to detect correlations between the AHI and other clinical variables including MSNAs. Based on the results of the univariate analysis, a multiple regression analysis with stepwise backward selection was performed to determine the contribution of clinical variables to AHI severity. A $p$-value of $\geq 0.05$ was an exclusion criteria for the stepwise procedure. In univariate and multivariate analysis, dummy variables are used in the analysis of nominal variables to include nominal variables into multivariate regression analysis. One-way ANOVA with Tukey's post-hoc test was used to analyze differences between groups. Pearson correlation coefficients were used to assess associations between study parameters. For all analyses, $p<0.05$ (two-sided) was considered statistically significant.

\section{RESULTS}

PSG measurements were performed for the first time in 186 OSAS patients at our sleep disorder lab (Sleep Disorders Laboratory of Kanazawa Municipal Hospital) from June 2012 to April 2014. Of these patients, multi-unit MSNA was performed in 148. Data from 46 patients were excluded because a singleunit MSNA reading could not be detected; this was due to a low signal-to-noise ratio. One hundred and two OSAS patients were finally included in this study. Characteristics of the study population are shown in Table 1. The study population consisted of $75 \%(n=77)$ males and $25 \%(n=25)$ females, with a mean age of $57 \pm 13$ years and mean body mass index (BMI) of $25.9 \pm 4.22 \mathrm{~kg} / \mathrm{m}^{2}$. The prevalence of hypertension was $57.8 \%$ ( $n=59) ; 8.82 \%(n=9)$ of patients had diabetes, and 29.4\% $(n=30)$ had dyslipidemia. The mean AHI was 34.5 \pm 21.4 events/h, mean BF was $54.8 \pm 12.2$ bursts $/$ min, mean BI was $81.1 \pm 13.0$ bursts/100 heartbeats, mean SF was 57.7 \pm 12.9 spikes/min, and mean SI was $85.0 \pm 17.1$ spikes/100 heartbeats.

\section{TABLE 1 | Baseline characteristics.}

\begin{tabular}{|c|c|}
\hline \multicolumn{2}{|l|}{ CLINICAL CHARACTERISTICS } \\
\hline Age (years) & $57 \pm 13$ \\
\hline Female/Male & $25 / 77$ \\
\hline BMI (kg/m2) & $25.9 \pm 4.22$ \\
\hline Hypertension (n, \%) & $59(57.8)$ \\
\hline Diabetes mellitus (n, \%) & $9(8.82)$ \\
\hline Dyslipidemia (n, \%) & $30(29.4)$ \\
\hline Systolic blood pressure (mmHg) & $132 \pm 16.5$ \\
\hline Diastolic blood pressure $(\mathrm{mmHg})$ & $81.3 \pm 12.2$ \\
\hline BF (bursts/min) & $54.8 \pm 12.2$ \\
\hline BI (bursts/100 heart beats) & $81.1 \pm 13.0$ \\
\hline SF (spikes/min) & $57.7 \pm 12.9$ \\
\hline SI (spikes/100 heart beats) & $85.0 \pm 17.1$ \\
\hline \multicolumn{2}{|l|}{ SLEEP PARAMETERS } \\
\hline $\mathrm{AHI}$ (events/h) & $34.5 \pm 21.4$ \\
\hline ESS & $8.14 \pm 4.98$ \\
\hline SREM (\%) & $17.3 \pm 6.79$ \\
\hline SES (\%) & $73.8 \pm 22.9$ \\
\hline Arousal index (events/h) & $38.3 \pm 20.7$ \\
\hline 3\%ODI (events/h) & $29.3 \pm 22.7$ \\
\hline Minimum SpO2 (\%) & $80.1 \pm 8.03$ \\
\hline Slow wave sleep (\%) & $3.58 \pm 7.02$ \\
\hline \multicolumn{2}{|l|}{ MEDICATION (n, \%) } \\
\hline ARB or ACEl & $33(32.4)$ \\
\hline Calcium antagonist & $30(29.4)$ \\
\hline$\beta$ blocker & $7(6.86)$ \\
\hline Diuretic & $5(4.90)$ \\
\hline Statin & $23(22.5)$ \\
\hline
\end{tabular}

Values are means $\pm S D$. BMI, Body mass index; $B F$, burst frequency; $B I$, burst incidence; $S F$, spike firing frequency; SI, spike firing incidence; $A H I$, apnea hypopnea index; ESS, Epworth sleepiness scale; SREM, Stage REM; SES, sleep efficiency score; ODI, $\mathrm{O}_{2}$ desaturation index; ARB, angiotensin II receptor blocker; ACEl, angiotensin converting enzyme inhibitor. 


\section{Relationship between AHI and Single- and Multi-Unit MSNA}

The correlation coefficients between AHI and each clinical parameter that may be affected by AHI tested by univariate and multivariate regression analyses are shown in Table 2. As expected, univariate analysis showed that multi-unit MSNA, expressed as BF and BI, was strongly correlated with AHI $(p<$ 0.001; Figure 2). Single-unit MSNA frequency and incidence were also significantly correlated with AHI. Interestingly, the coefficients were higher between AHI and single-unit MSNA than between AHI and multi-unit MSNA. In addition, BMI and systolic and diastolic blood pressure were correlated with AHI. However, as shown in Table 2, multivariate analysis with stepwise method revealed that SF, BMI, and diastolic blood pressure were associated with AHI; however, SF had the most strongly significant relationship with AHI $(T=7.27, p<0.0001)$.

\section{Comparison of Baseline Characteristics According to AHI}

Patients were divided into three groups according to AHI. The least severe group (S) comprised patients with an AHI of 5-30 events/h, the severe OSAS (SS) group comprised patients with an AHI of 30-55 events/h, and the very severe OSAS (VSS) group comprised patients with $>55$ events/h. Patient characteristics for each group are shown in Table 3. There was no significant difference in age among the three groups. The VSS and SS groups had more males than the $S$ group. The VSS group had the highest
TABLE 2 | Regression analysis between AHI and clinical parameters.

\begin{tabular}{|c|c|c|c|c|c|c|}
\hline \multicolumn{7}{|c|}{ AHI } \\
\hline \multicolumn{3}{|c|}{$\begin{array}{c}\text { Univariate regression } \\
\text { analysis }\end{array}$} & \multicolumn{4}{|c|}{$\begin{array}{l}\text { Multivariate regression } \\
\text { analysis }\end{array}$} \\
\hline & $R$ & $P$ & $\beta$ & $T$ & $P$ & VIF \\
\hline $\mathrm{BF}$ & 0.437 & $<0.0001$ & & & & \\
\hline SF & 0.632 & $<0.0001$ & 0.479 & 7.27 & $<0.0001$ & 1.13 \\
\hline $\mathrm{BMI}$ & 0.580 & $<0.0001$ & 0.360 & 5.37 & $<0.0001$ & 1.17 \\
\hline DM & 0.192 & 0.052 & & & & \\
\hline $\mathrm{DL}$ & 0.128 & 0.200 & & & & \\
\hline Gender & 0.217 & 0.029 & & & & \\
\hline Age & 0.084 & 0.399 & & & & \\
\hline SBP & 0.370 & $<0.0001$ & & & & \\
\hline DBP & 0.408 & $<0.0001$ & 0.276 & 4.35 & $<0.0001$ & 1.05 \\
\hline
\end{tabular}

AHI, apnea hypopnea index; BF, burst frequency (bursts/ min); BMI, Body mass index $\left(\mathrm{kg} / \mathrm{m}^{2}\right)$; $D M$, diabetes mellitus (with or without); $D L$, dyslipidemia (with or without); SBP, systolic blood pressure $(\mathrm{mmHg})$; DBP, diastolic blood pressure $(\mathrm{mmHg})$; VIF, variance inflation factor. $\beta$-values show standardized regression coefficients. T-values mean $t$ statistic.

BMI, which was significantly different from those of both the SS and $S$ groups. As predicted, sleep parameters were significantly worse in both the VSS and SS groups than in the S group. In addition, systolic and diastolic blood pressure were significantly higher in the VSS and SS groups than in the S group. The proportion of patients treated with antihypertensive drugs and/or statins were similar among the three groups.
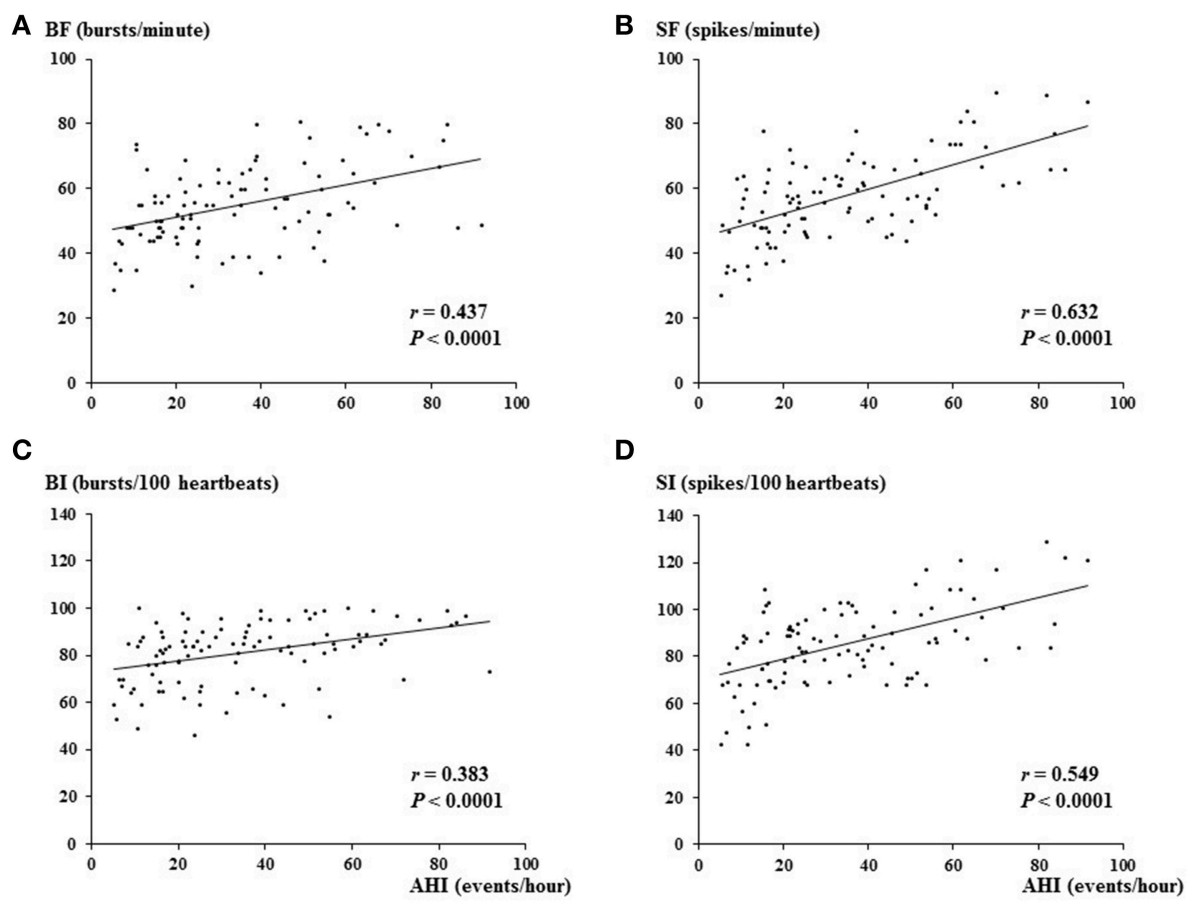

FIGURE 2 | Relationship between AHI and multi-unit MSNA (A, burst frequency; C, burst incidence) or single-unit MSNA (B, spike frequency; D, spike incidence). The correlation coefficient between single-unit MSNA and AHI was stronger than that between multi-unit MSNA and AHI. AHI, apnea-hypopnea index; $\mathrm{BF}$, burst frequency; SF, spike frequency; BI, burst incidence; SI, spike incidence. 


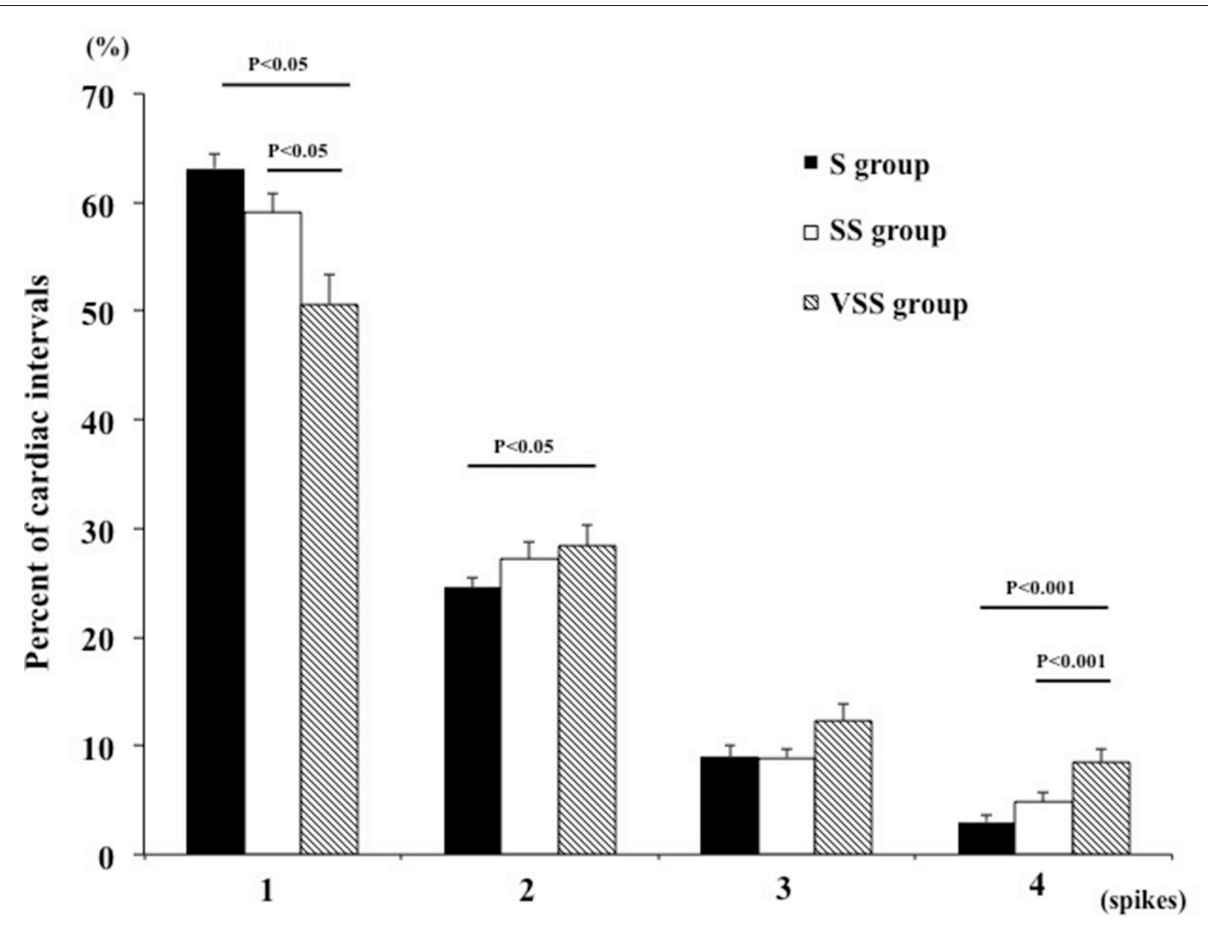

FIGURE 3 | Percentages of cardiac intervals in which one, two, three, or four single-unit spikes were calculated separately. In the VSS group, there was a significant decrease in the percentage of cardiac intervals that contained one spike compared with the other groups, and a significant increase was observed in the proportion of cardiac intervals that had four spikes compared with the other groups. There were no statistical differences in the firing properties of the S and SS groups. S group, AHI of 5-30 events/h; SS group, AHI of 30-55 events/h; VSS group, AHI of >55 events/h. AHI, apnea-hypopnea index.

\section{Differences in Single- and Multi-Unit MSNA among the Three Groups}

Both single- and multi-unit MSNA were significantly augmented in the VSS group compared with the SS and S groups, as shown Table 4. The same results were observed in the assessment of single- and multi-unit MSNA incidents. As shown in Table 4, the firing probability of single-unit MSNA in the SS and VSS group was significantly augmented compared with the $S$ group. The multiple spike incidence in the VSS group was significantly augmented compared with the other groups. Figure 3 shows the distribution of cardiac intervals in which there were one, two, three, or four single-unit spikes firing. In the VSS group, there was a significant decrease in the percentage of cardiac intervals that contained one spike compared with the other groups. The distribution of cardiac intervals in which two and three spikes occurred tended to increase in the VSS group. Moreover, significant augmentation was observed in the proportion of cardiac intervals that had four spikes in the VSS group compared with other groups. However, the distribution of cardiac intervals according to spike firings was similar between the S and SS groups.

\section{DISCUSSION}

This is the first report to examine the relationship between AHI and single- and multi-unit MSNA in OSAS patients. The novel and important findings from this study are that (1)
BMI, blood pressure, and single- and multi-unit MSNA were significantly associated with AHI, but single-unit MSNA was the most significantly correlated with AHI as determine by multivariate regression analysis and (2) single- and multi-unit MSNA increased according to AHI severity. However, patients with an AHI of $>55$ events/h had a higher proportion of multiple firing spikes than did the other groups, although the results from patients with an AHI of 30-55 events/h were not significantly different than the patients with an AHI of $<30$ events/h. These results suggest that in intense sympathoexcitation states like severe OSAS, single-unit MSNA is useful to evaluate sympathetic outflow to the periphery.

\section{Classification of OSAS Severity and Sympathetic Nerve Activity}

Generally, sleep apnea is classified as no sleep apnea (AHI 515), mild OSA (AHI 15-30), moderate OSA, and severe OSA (AHI > 30; The Report of an American Academy of Sleep Medicine Task Force, 1999). Previous studies have demonstrated that OSAS mortality is increased in patients with an AHI of $>30$ events/h, and this can partly be attributed to augmented sympathetic activity (Chesson et al., 2003; Gami et al., 2005). In this study, multi-unit MSNA increased with AHI severity. Multi-unit MSNA is a reliable method with which to assess sympathetic nerve activity in humans, and augmented multi-unit MSNA has been shown to correlate with mortality in HF patients (Barretto et al., 2009). High sympathetic nerve activity is a leading 
TABLE 3 | Comparison of baseline characteristics according to OSAS severity.

\begin{tabular}{|c|c|c|c|}
\hline Events/h & S: $5 \sim 30(n=52)$ & SS: $30 \sim 55(n=32)$ & VSS: $55 \sim(n=18)$ \\
\hline \multicolumn{4}{|c|}{ CLINICAL CHARACTERISTICS } \\
\hline Age & $57 \pm 14$ & $61 \pm 10$ & $52 \pm 13$ \\
\hline Male (n, \%) & $18(34.6)$ & $27(84.4)^{\star}$ & $15(83.3)^{\star}$ \\
\hline $\mathrm{BMl}(\mathrm{kg} / \mathrm{m} 2)$ & $23.9 \pm 3.17$ & $27.0 \pm 4.01$ & $29.5 \pm 4.27^{\star \#}$ \\
\hline Hypertension (n, \%) & $28(53.8)$ & $20(62.5)$ & $11(61.1)$ \\
\hline $\begin{array}{l}\text { Diabetes mellitus ( } \mathrm{n} \text {, } \\
\%)\end{array}$ & $1(1.92)$ & $5(15.6)^{\star}$ & $3(16.7)$ \\
\hline Dyslipidemia (n, \%) & $17(32.7)$ & $10(31.3)$ & $3(16.7)$ \\
\hline $\begin{array}{l}\text { Systolic blood } \\
\text { pressure (mmHg) }\end{array}$ & $126 \pm 15.4$ & $135.3 \pm 14.1^{\star}$ & $142.5 \pm 17.9^{*}$ \\
\hline $\begin{array}{l}\text { Diastolic blood } \\
\text { pressure }(\mathrm{mmHg})\end{array}$ & $77.3 \pm 10.6$ & $84.3 \pm 10.6^{\star}$ & $87.6 \pm 15.2^{\star}$ \\
\hline \multicolumn{4}{|c|}{ SLEEP PARAMETERS } \\
\hline AHI (events/h) & $17.4 \pm 6.67$ & $42.5 \pm 7.51^{*}$ & $70.0 \pm 11.1^{\star \#}$ \\
\hline ESS & $8.10 \pm 5.05$ & $7.31 \pm 4.75$ & $9.72 \pm 5.06^{\star}$ \\
\hline SREM (\%) & $18.2 \pm 7.45$ & $16.7 \pm 4.60$ & $15.6 \pm 7.94$ \\
\hline SES (\%) & $78.0 \pm 26.3$ & $70.9 \pm 16.6^{\star}$ & $66.8 \pm 20.2^{\star}$ \\
\hline $\begin{array}{l}\text { Arousal index } \\
\text { (events/h) }\end{array}$ & $23.8 \pm 8.96$ & $43.0 \pm 11.2^{*}$ & $71.9 \pm 13.9^{\star \#}$ \\
\hline 3\% ODI (events/h) & $12.2 \pm 6.93$ & $36.3 \pm 11.2^{*}$ & $66.5 \pm 15.5^{\star \#}$ \\
\hline Minimum SpO2 (\%) & $83.9 \pm 5.29$ & $78.6 \pm 6.92^{\star}$ & $71.6 \pm 9.19^{\star \#}$ \\
\hline \multicolumn{4}{|l|}{ MEDICATION (n, \%) } \\
\hline ARB or ACEl & 19 (36.5) & $9(28.1)$ & $5(27.8)$ \\
\hline Calcium antagonist & $14(26.9)$ & $12(37.5)$ & $4(22.2)$ \\
\hline$\beta$ blocker & $3(5.77)$ & $3(9.38)$ & $1(5.56)$ \\
\hline Diuretic & $1(1.92)$ & $3(9.38)$ & $1(5.56)$ \\
\hline Statin & 15 (28.8) & $4(12.5)$ & $4(22.2)$ \\
\hline
\end{tabular}

Values are means $\pm S D$. BMI, Body mass index; $B F$, burst frequency; Bl, burst incidence; $S F$, spike firing frequency; SI, spike firing incidence; $A H$, apnea hypopnea index; ESS, Epworth sleepiness scale; SREM, Stage REM; SES, sleep efficiency score; ODI, $\mathrm{O}_{2}$ desaturation index; $A R B$, angiotensin II receptor blocker; ACEl, angiotensin converting enzyme inhibitor. ${ }^{*} p<0.05$, compared to $S$ group; ${ }^{*} p<0.05$, compared to SS group.

cause of cardiovascular diseases and can contribute to higher mortality in patients with HF as well as chronic kidney disease (Penne et al., 2009). In addition, we found that the probability of multiple firings in single-unit MSNA was significantly increased in patients with an AHI score $>30$ events/h (Table 4). Lambert et al. (2011) observed that the incidence of multiple firing in single-unit MSNA was associated with cardiac noradrenaline spillover in humans. These results suggest that high mortality in OSAS patients with an AHI of $>30$ events/h may be attributed to the increased probability of multiple spikes firing during one cardiac interval.

The results also showed that the distribution of each multiple spike was significantly augmented in patients with high AHIs (>55 events/h) compared with other OSAS patients. There were two reasons to determine the AHI cutoff of 55 events/h. One was that the mean AHI + standard deviation in this study was 55.9 events/h. Another was that the percentage of four spikes firing within one cardiac interval in patients with an AHI of $>55$ events/h was significantly higher than that in patients with an AHI of $<55$ events/h $(p=0.02)$. However, patients with
TABLE 4 | Comparison of sympathetic nerve activity according to OSAS severity.

\begin{tabular}{lccc}
\hline & S & SS & vSS \\
\hline MULTI-UNIT MSNA & & & \\
BF (bursts/min) & $50.6 \pm 9.71$ & $56.3 \pm 12.6^{\star}$ & $64.6 \pm 12.0^{\star \#}$ \\
BI (bursts/100 heart beats) & $77.4 \pm 12.9$ & $82.6 \pm 13.2$ & $89.2 \pm 8.57^{\star \#}$ \\
SINGLE-UNIT MSNA & & & \\
SF (spikes/min) & $51.5 \pm 10.8$ & $59.0 \pm 8.85^{\star}$ & $73.2 \pm 10.9^{\star \#}$ \\
SI (spikes/100 heart beats) & $78.4 \pm 15.6$ & $86.5 \pm 13.6^{\star}$ & $101 \pm 15.7^{\star \#}$ \\
Firing probability (\%) & $50.4 \pm 10.8$ & $55.4 \pm 9.67^{\star}$ & $60.4 \pm 10.1^{\star}$ \\
Multiple spike incidence (\%) & $37.5 \pm 10.1$ & $40.9 \pm 10.2^{\star}$ & $49.2 \pm 11.2^{\star \#}$ \\
\hline
\end{tabular}

Values are means $\pm S D$. S, SAS group; SS, severe SAS group; VSS, very severe SAS group; BF, burst frequency (bursts/min); BI, burst incidence (bursts/100 heart beats); $S F$, spike firing frequency (spikes/min); SI, spike firing incidence (spikes/100 heart beats). ${ }^{*} p<0.05$, compared to $S$ group; ${ }^{*} p<0.05$, compared to SS group.

an AHI of $>54$ events/h were not significantly different from those with an AHI of $<54$ events/h $(p=0.07)$. These results suggest that patients with high AHIs ( $>55$ events/h) have a high sympathoexcitation state, which may contribute to higher mortality.

\section{Assessment of Single-Unit MSNA in OSAS Patients}

Previous studies, including our own, have reported that singleunit MSNA is a more accurate and reliable assessment of sympathetic nerve activity in both healthy and diseased patients (Macefield et al., 1999; Hogarth et al., 2011; Murai et al., 2012; Hering et al., 2013; Millar et al., 2015). Multi-unit MSNA is limited in that it cannot exceed heart rate, and because it is mostly regulated by arterial baroreceptors, burst occurrence is synchronized with cardiac interval. Single-unit MSNA can assess the multiple firings of individual muscle vasoconstrictor neurons during one cardiac interval, and it is not restricted by heart rate. The most important advantage of single-unit MSNA analysis is the ability to demonstrate multiple spikes firing within one cardiac interval. Single-unit MSNA reveals the sympathetic firing mechanism to the periphery. The first mechanism is an increase in the firing frequency of vasoconstrictor fibers that are already active. The second mechanism is an increase in multiple firing within one cardiac interval. The last mechanism is the recruitment of previously silent fibers. Increased multiple firing incidents within one cardiac interval is a state of intense sympathoexcitation in both healthy subjects (in the Valsalva maneuver) and HF patients (Murai et al., 2012). Elam et al. (2002) reported that in eight OSAS patients, the single-unit MSNA frequency exceeded multi-unit MSNA during one cardiac interval. These findings are consistent with our results in that the number of multiple firing spikes within one cardiac interval increased in very severe OSAS patients. In addition to previous findings, we demonstrated that increased firing characteristics of single-unit MSNA were correlated with AHI severity. Interestingly, our data showed that the distribution of each spike firing did not differ between patients with an AHI of $<30$ events/h and those with an AHI of 30-55 events/h. However, 
the single-unit MSNA frequency, incidence, and multiple firing incidence were significantly higher in patients with an AHI of 3055 events/h than in patients with an AHI of $<30$ events/h. These findings suggest that the mechanism of the increase in multiple single-unit sympathetic firing within one cardiac interval may be maintained until the patient reaches a state of very severe OSAS. In fact, our data showed that the patients with an AHI of $>55$ events/h had significantly higher blood pressure than the less severe groups.

\section{Clinical Implications}

The augmentation of multi-unit MSNA in OSAS patients, both during sleep and waking hours, is well established. The relationship between OSAS severity and sympathoexcitation is poorly understood, and the underlying mechanism responsible for sympathetic nerve activity in OSAS remains unclear. However, our results strongly suggest that long-term, apneainduced hypoxia can contribute to increased sympathetic nerve activity. Several studies have demonstrated that continuous positive airway pressure (CPAP) treatment improves left ventricular (LV) function in OSAS patients (Kaneko et al., 2003) and inhibits sympathetic nerve activity (measured by MSNA or overnight urinary norepinephrine excretion). CPAP treatment has also been reported to lower norepinephrine levels (Bradley et al., 2005) and reduce risk after heart transplantation (Arzt et al., 2007); however, not all studies have shown improved LV function (Somers et al., 2008). The results from this study suggest that the reduction in AHI by CPAP treatment is not necessarily accompanied by the inhibition of single-unit MSNA firing. Greenwood et al. (2001) demonstrated that single-unit MSNA frequency is associated with LV hypertrophy. In some OSAS patients, MSNA may not be inhibited by CPAP treatment even though the AHI is reduced; in these cases, multi-unit MSNA underestimates sympathetic firing in very severe OSAS patients. Further studies involving more patients are needed to confirm the effects of CPAP on the single-unit MSNA response to LV function and mortality.

\section{Limitations}

This study has some limitations. First, the sampling size in this study was relatively large, but confounding factors still need to be considered. However, this study had an extremely large sampling size compared with other studies. Second, most of the patients (>50\%) had been previously treated with antihypertensive drugs

\section{REFERENCES}

Arzt, M., Floras, J. S., Logan, A. G., Kimoff, R. J., Series, F., Morrison, D., et al. (2007). Suppression of central sleep apnea by continuous positive airway pressure and transplant-free survival in heart failure: a post hoc analysis of the Canadian Continuous Positive Airway Pressure for Patients with Central Sleep Apnea and Heart Failure Trial (CANPAP). Circulation 115, 3173-3180. doi: 10.1161/CIRCULATIONAHA.106.683482

Barretto, A. C., Santos, A. C., Munhoz, R., Rondon, M. U., Franco, F. G., Trombetta, I. C., et al. (2009). Increased muscle sympathetic nerve activity predicts mortality in heart failure patients. Int. J. Cardiol. 135, 302-307. doi: 10.1016/j.ijcard.2008.03.056 such as angiotensin converting enzymes, angiotensin receptor blockers, calcium channel blockers, or thiazide diuretics. These drugs may affect sympathetic nerve activity, and in fact, some antihypertensive drugs have been reported to inhibit sympathetic nerve activity. However, even in patients treated with these drugs, sympathetic nerve activity was still associated with sleep apnea severity, although the proportion of the patients treated with antihypertensive drugs and/or statins were similar among the three groups. In addition, there have been no reports about these effects on MSNA in OSAS patients, except for CPAP. Our results showed that single-unit MSNA was still higher in severe OSAS patients treated with antihypertensive drugs. Lastly, this study was a cross-sectional study that did not include any morbidity or mortality endpoints. Further studies are needed to elucidate the interaction between single-unit MSNA and cardiovascular mortality in OSAS patients.

\section{CONCLUSION}

In this study, we found that single- and multi-unit MSNA was significantly associated with OSAS severity. Moreover, singleunit MSNA was the most significantly correlated with AHI. The multiple firing spikes ratio measure by single-unit MSNA was significantly higher in very severe OSAS patients. These results suggest that single-unit MSNA is a more accurate reflection of sleep apnea severity and firing property alterations, especially in patients with very severe OSAS.

\section{AUTHOR CONTRIBUTIONS}

TH, HM: Substantial contribution to the conception or design of the work. HT, YS, YN, YO, TK, SU, TH: Acquisition, analysis, or interpretation of data for work. HM, SK, HF: Revising the work critically for important intellectual content. MT: Final approval of the revision to be published. SK, ST, HM: Agreement to be accountable for all aspects of the work in ensuring that questions related to the accuracy or integrity of any part of the work are appropriately investigated and resolved.

\section{ACKNOWLEDGMENTS}

This work was supported by JSPS KAKENHI Grant-in-Aid for Scientific Research (C) Number 26350497. 
Chesson, A. L. Jr., Berry, R. B., and Pack, A. (2003). Practice parameters for the use of portable monitoring devices in the investigation of suspected obstructive sleep apnea in adults. Sleep 26, 907-913.

Cooper, V. L., Elliott, M. W., Pearson, S. B., Taylor, C. M., Mohammed, M. M., and Hainsworth, R. (2007). Daytime variability of baroreflex function in patients with obstructive sleep apnoea: implications for hypertension. Exp. Physiol. 92, 391-398. doi: 10.1113/expphysiol.2006.035584

Cooper, V. L., Pearson, S. B., Bowker, C. M., Elliott, M. W., and Hainsworth, R. (2005). Interaction of chemoreceptor and baroreceptor reflexes by hypoxia and hypercapnia - a mechanism for promoting hypertension in obstructive sleep apnoea. J. Physiol. 568, 677-687. doi: 10.1113/jphysiol.2005.094151

Elam, M., McKenzie, D., and Macefield, V. (2002). Mechanisms of sympathoexcitation: single-unit analysis of muscle vasoconstrictor neurons in awake OSAS subjects. J. Appl. Physiol. (1985) 93, 297-303. doi: 10.1152/japplphysiol.00899.2001

Gami, A. S., Howard, D. E., Olson, E. J., and Somers, V. K. (2005). Day-night pattern of sudden death in obstructive sleep apnea. N. Engl. J. Med. 352, 1206-1214. doi: 10.1056/NEJMoa041832

Greenwood, J. P., Scott, E. M., Stoker, J. B., and Mary, D. A. (2001). Hypertensive left ventricular hypertrophy: relation to peripheral sympathetic drive. J. Am. Coll. Cardiol. 38, 1711-1717. doi: 10.1016/S0735-1097(01)01600-X

Hausberg, M., Kosch, M., Harmelink, P., Barenbrock, M., Hohage, H., Kisters, K., et al. (2002). Sympathetic nerve activity in end-stage renal disease. Circulation 106, 1974-1979. doi: 10.1161/01.CIR.0000034043.16664.96

Hering, D., Lambert, E. A., Marusic, P., Walton, A. S., Krum, H., Lambert, G. W., et al. (2013). Substantial reduction in single sympathetic nerve firing after renal denervation in patients with resistant hypertension. Hypertension 61, 457-464. doi: 10.1161/HYPERTENSIONAHA.111.00194

Hogarth, A. J., Graham, L. N., Corrigan, J. H., Deuchars, J., Mary, D. A., and Greenwood, J. P. (2011). Sympathetic nerve hyperactivity and its effect in postmenopausal women. J. Hypertens. 29, 2167-2175. doi: 10.1097/HJH.0b013e32834b8014

Hogarth, A. J., MacKintosh, A. F., and Mary, D. A. (2006). The sympathetic drive after acute myocardial infarction in hypertensive patients. Am. J. Hypertens. 19, 1070-1076. doi: 10.1016/j.amjhyper.2006.03.015

Hunt, S. A., Abraham, W. T., Chin, M. H., Feldman, A. M., Francis, G. S., Ganiats, T. G., et al. (2009). 2009 focused update incorporated into the ACC/AHA 2005 guidelines for the diagnosis and management of heart failure in adults: a report of the American College of Cardiology Foundation/American Heart Association Task Force on Practice Guidelines: developed in collaboration with the International Society for Heart and Lung Transplantation. Circulation 119, e391-e479. doi: 10.1016/j.jacc.2008.11.013

Ikeda, T., Murai, H., Kaneko, S., Usui, S., Kobayashi, D., Nakano, M., et al. (2012). Augmented single-unit muscle sympathetic nerve activity in heart failure with chronic atrial fibrillation. J. Physiol. 590, 509-518. doi: 10.1113/jphysiol.2011.223842

Kaneko, Y., Floras, J. S., Usui, K., Plante, J., Tkacova, R., Kubo, T., et al. (2003). Cardiovascular effects of continuous positive airway pressure in patients with heart failure and obstructive sleep apnea. N. Engl. J. Med. 348, 1233-1241. doi: 10.1056/NEJMoa022479

Lambert, E. A., Schlaich, M. P., Dawood, T., Sari, C., Chopra, R., Barton, D. A., et al. (2011). Single-unit muscle sympathetic nervous activity and its relation to cardiac noradrenaline spillover. J. Physiol. 589, 2597-2605. doi: 10.1113/jphysiol.2011.205351

Leuenberger, U., Jacob, E., Sweer, L., Waravdekar, N., Zwillich, C., and Sinoway, L. (1995). Surges of muscle sympathetic nerve activity during obstructive apnea are linked to hypoxemia. J. Appl. Physiol. (1985) 79, 581-588.
Macefield, V. G., Rundqvist, B., Sverrisdottir, Y. B., Wallin, B. G., and Elam, M. (1999). Firing properties of single muscle vasoconstrictor neurons in the sympathoexcitation associated with congestive heart failure. Circulation 100, 1708-1713. doi: 10.1161/01.CIR.100.16.1708

Millar, P. J., Murai, H., and Floras, J. S. (2015). Paradoxical muscle sympathetic reflex activation in human heart failure. Circulation 131, 459-468. doi: 10.1161/CIRCULATIONAHA.114.010765

Mooe, T., Rabben, T., Wiklund, U., Franklin, K. A., and Eriksson, P. (1996). Sleepdisordered breathing in men with coronary artery disease. Chest 109, 659-663. doi: 10.1378/chest.109.3.659

Morgan, B. J., Crabtree, D. C., Palta, M., and Skatrud, J. B. (1995). Combined hypoxia and hypercapnia evokes long-lasting sympathetic activation in humans. J. Appl. Physiol. (1985) 79, 205-213.

Murai, H., Takamura, M., and Kaneko, S. (2012). Advantage of recording singleunit muscle sympathetic nerve activity in heart failure. Front. Physiol. 3:109. doi: 10.3389/fphys.2012.00109

Murai, H., Takamura, M., Maruyama, M., Nakano, M., Ikeda, T., Kobayashi, D., et al. (2009). Altered firing pattern of single-unit muscle sympathetic nerve activity during handgrip exercise in chronic heart failure. J. Physiol. 587, 2613-2622. doi: 10.1113/jphysiol.2009.172627

Murai, H., Takata, S., Maruyama, M., Nakano, M., Kobayashi, D., Otowa, K., et al. (2006). The activity of a single muscle sympathetic vasoconstrictor nerve unit is affected by physiological stress in humans. Am. J. Physiol. Heart Circ. Physiol. 290, H853-H860. doi: 10.1152/ajpheart.00184.2005

Penne, E. L., Neumann, J., Klein, I. H., Oey, P. L., Bots, M. L., and Blankestijn, P. J. (2009). Sympathetic hyperactivity and clinical outcome in chronic kidney disease patients during standard treatment. J. Nephrol. 22, 208-215.

Somers, V. K., White, D. P., Amin, R., Abraham, W. T., Costa, F., Culebras, A., et al. (2008). Sleep apnea and cardiovascular disease: an American Heart Association/american College Of Cardiology Foundation Scientific Statement from the American Heart Association Council for High Blood Pressure Research Professional Education Committee, Council on Clinical Cardiology, Stroke Council, and Council On Cardiovascular Nursing. In collaboration with the National Heart, Lung, and Blood Institute National Center on Sleep Disorders Research (National Institutes of Health). Circulation 118, 1080-1111. doi: 10.1161/CIRCULATIONAHA.107.189420

The Report of an American Academy of Sleep Medicine Task Force (1999). Sleep-related breathing disorders in adults: recommendations for syndrome definition and measurement techniques in clinical research. Sleep 22, 667-689.

Vallbo, A. B., Hagbarth, K. E., Torebjörk, H. E., and Wallin, B. G. (1979) Somatosensory, proprioceptive, and sympathetic activity in human peripheral nerves. Physiol. Rev. 59, 919-957.

Young, T., Finn, L., Peppard, P. E., Szklo-Coxe, M., Austin, D., Nieto, F. J., et al. (2008). Sleep disordered breathing and mortality: eighteen-year follow-up of the Wisconsin sleep cohort. Sleep 31, 1071-1078.

Conflict of Interest Statement: The authors declare that the research was conducted in the absence of any commercial or financial relationships that could be construed as a potential conflict of interest.

Copyright (C) 2016 Hamaoka, Murai, Kaneko, Usui, Okabe, Tokuhisa, Kato, Furusho, Sugiyama, Nakatsumi, Takata and Takamura. This is an open-access article distributed under the terms of the Creative Commons Attribution License (CC BY). The use, distribution or reproduction in other forums is permitted, provided the original author(s) or licensor are credited and that the original publication in this journal is cited, in accordance with accepted academic practice. No use, distribution or reproduction is permitted which does not comply with these terms. 